\title{
Polarization Switching of a Vertical-Cavity Semiconductor Laser as a Kramers Hopping Problem
}

\author{
M. B. Willemsen, M. U. F. Khalid, M. P. van Exter, and J.P. Woerdman \\ Huygens Laboratory, Leiden University, P.O. Box 9504, 2300 RA Leiden, The Netherlands
}

(Received 30 November 1998)

\begin{abstract}
We report stochastic polarization switching in vertical-cavity semiconductor lasers, with residence times that vary by 8 orders of magnitude for a single such laser by changing its switch current with a hot-spot technique. In spite of the potentially complicated polarization dynamics of these lasers, the experimental results agree with Kramers hopping in a 1D double-well potential initiated by quantum fluctuations. We confirm the validity of this surprisingly simple theoretical model by independent measurements of the potential barrier between the wells and the spontaneous emission noise strength. [S0031-9007(99)09390-4]
\end{abstract}

PACS numbers: 42.65.Pc, 42.55.Px

The vertical cavity surface emitting laser (VCSEL) is a novel type of semiconductor laser, which is not completely understood. This refers, in particular, to important issues such as polarization stability, polarization switching, and polarization modulation. It is generally believed that polarization behavior of VCSELs is extremely complicated, in particular, when considering multitransversemode devices [1]. However, recently a greatly simplified theory was developed, which allows analytical solutions and which predicts that the polarization dynamics of VCSELs reduces to the Kramers hopping problem of a 1D double-well potential [2]. We note that the Kramers double-well potential model has a long history [3] and has been applied in many subfields of physics and chemistry [4]. In this Letter we validate this surprisingly simple theory for practical VCSELs by observing and analyzing stochastic polarization switching.

The important quantity in the Kramers model is the average residence time $\langle T\rangle$ (also called average dwell time [2] or first-passage time [4]), which is the average time before a switch takes place; $\langle T\rangle$ depends on the potential barrier between the wells and the strength of the quantum fluctuations that initiate switching. The experimental validation of the analytical VCSEL theory [2] has become possible by using a hot-spot technique; this has allowed us to manipulate the Kramers potential of a single VCSEL. In this way we have been able to change the average residence time $\langle T\rangle$ by 8 orders of magnitude, in quantitative agreement with theory.

The reason for this dramatic change in residence times is that a VCSEL has a rather critical balance between the deterministic force that pins the polarization and the stochastic force due to quantum fluctuations (spontaneous emission) that triggers switching. The anisotropies which determine the stationary polarization are relatively small, because of the nominal cylindrical symmetry of the cavity, and the spontaneous emission noise is relatively large due to the small size of the device [2].

As the underlying theory has already been discussed [2], we will only highlight its essentials. The start- ing point is our recent theoretical and experimental work $[2,5,6]$ showing that the polarization dynamics of quantum-well VCSELs, as derived from a split-level inversion model [7-9], after adiabatic elimination of the spin-difference inversion is similar to that of a class A-type (gas or dye) laser when the anisotropies are not too big. In this description the polarization, described by the polarization angle $(\phi)$ and ellipticity angle $(\chi)$, is driven by dispersive and absorptive anisotropies. Specifically, the effective birefringence $\left(\omega_{\text {eff }}\right)$ and effective dichroism $\left(\gamma_{\text {eff }}\right)$ create a difference in frequency and gain between the lasing and nonlasing polarization, respectively. Both $\omega_{\text {eff }}$ and $\gamma_{\text {eff }}$ consist of a linear part, which quantifies how much the cylindrical symmetry of the cavity is broken, and a nonlinear part, which corresponds to a polarization dependence of the optical saturation and is thus proportional to the pump parameter $\mu=\left(I / I_{\mathrm{th}}\right)-1$. When linear birefringence is the dominant anisotropy (which is the case for practical VCSELs $[2,5,6]$ ), we have

$$
\begin{gathered}
\omega_{\mathrm{eff}}=\sqrt{\omega_{\text {lin }}^{2}+2 \omega_{\text {lin }} \omega_{\text {non }}-\gamma_{\text {non }}^{2}}, \\
\gamma_{\mathrm{eff}}=\gamma_{\text {lin }} \cos (2 \beta)+\gamma_{\mathrm{non}}, \\
\omega_{\text {non }}=\alpha \gamma_{\text {non }}=\alpha \frac{\kappa}{\Gamma} \mu,
\end{gathered}
$$

where $\omega_{\text {lin }}$ and $\omega_{\text {non }}$ are, respectively, the linear and nonlinear birefringence, $\gamma_{\text {lin }}$ and $\gamma_{\text {non }}$ are, respectively, the linear and nonlinear dichroism, $\kappa$ is the decay rate of the intracavity field, $\Gamma$ is the decay rate of the spin-difference inversion divided by that of the spin-averaged inversion, and $\alpha$ is the phase-amplitude coupling factor. The angle $\beta$, between the axes of the linear birefringence and linear dichroism, is introduced to describe nonaligned linear anisotropies. For $\beta \neq 0$ the effect of linear dichroism is reduced, because the linear dichroism must be projected onto the axis of the linear birefringence, since linear birefringence has been assumed to be the dominant anisotropy.

Besides the deterministic force due to anisotropies, which sets the steady-state polarization, there is also 
a stochastic force due to quantum fluctuations. The presence of spontaneous emission results in a "noise cloud" around the steady-state polarization $(\phi, \chi)$ on the Poincaré sphere (Fig. 1). (On the Poincaré sphere the equator corresponds to all states of linear polarization, the poles to the two states of circular polarization, and the rest to elliptically polarized light.)

In general, the fluctuations in $\phi$ and $\chi$ can be quite different. However, for practical VCSELs, where the linear birefringence is the dominant anisotropy, this difference in fluctuations of both angles is negligible and the polarization dynamics can be reduced to that of single angle $\varphi$, where $\cos (2 \varphi)=\cos (2 \chi) \cos (2 \phi)$ [2]. The very complicated polarization dynamics of VCSELs is then reduced to the standard model of diffusion in a onedimensional potential. To obtain more insight into the statistics we derive from the Fokker-Planck equation for the polarization [2] as steady-state probability density

$$
\begin{gathered}
P(\varphi) d \varphi \propto \sin (2 \varphi) e^{-V(\varphi) / D} d \varphi, \\
V(\varphi)=-\frac{\gamma_{\operatorname{lin}} \cos (2 \beta)}{2} \cos (2 \varphi)-\frac{\gamma_{\mathrm{non}}}{8} \cos (4 \varphi) .
\end{gathered}
$$

The parameter $D$ is the strength of the Langevin noise source that models spontaneous emission. We will now limit ourselves to a symmetric double-well potential, where the modes are equivalent $\left[\gamma_{\text {lin }} \cos (2 \beta)=0\right]$. The potential has in fact two series of minima, at $2 \varphi=0$ $[(\bmod 2 \pi)$ and $2 \varphi=\pi(\bmod 2 \pi)$, which corresponds to pure $x$ - and $y$-polarized emission. Noise can make the system switch between two neighboring minima. The evolution of the two polarization angles $\phi$ and $\chi$ on the Poincare sphere during a switch is shown in Fig. 1. The switching occurs between two orthogonally linear polarized states, which lie diametrically in the equatorial plane. The trajectory which connects the polarization before and after the switch is a spiraling motion because

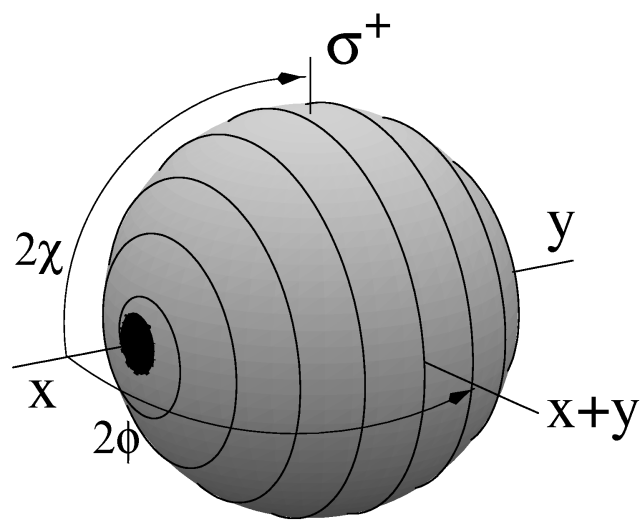

FIG. 1. Evolution of the polarization angles $\chi$ and $\phi$ during the switch, plotted on the Poincaré sphere. The switch corresponds to a spiraling motion between two linear polarizations, diametrically opposed in the equatorial plane. The black dot indicates the noise cloud. we assumed the linear birefringence to be dominant. The angle $2 \varphi$ measures the azimuthal position of the almost circular orbits around the $x, y$ axis.

Straightforward calculation yields an exponential distribution of residence times (for $\gamma_{\text {non }} \gg D$, which is satisfied in practice; see below), with an average residence time of each eigenpolarization given by

$$
\langle T\rangle \approx \sqrt{\frac{\pi D}{\gamma_{\text {non }}}} \frac{1}{\gamma_{\text {non }}} \exp \left(\frac{\gamma_{\text {non }}}{4 D}\right) .
$$

Since the potential is symmetric, $\langle T\rangle$ is the same for both linear polarizations, i.e., the laser switches symmetrically. The rate of switching is thus expressed in terms of the nonlinear dichroism $\gamma_{\mathrm{non}}$, which specifies the barrier between the two potential wells, and the diffusion coefficient $D$. The exponent in Eq. (3) depends quadratically on the pump parameter $\mu$, because the potential barrier scales with the pump $\left(\gamma_{\mathrm{non}} \propto \mu\right)$, due to polarization-dependent saturation, and $D \propto 1 / \mu$ (Schawlow-Townes). This "quadratic exponential" dependence makes the residence time a very sensitive function of the pump parameter.

For the experiments we had a batch of about 50 proton implanted $\mathrm{Al}_{x} \mathrm{Ga}_{1-x} \mathrm{As}$ VCSELs available, operating at $850 \mathrm{~nm}$ [10]. Within the range of fundamental tranverse mode operation, between $I_{\mathrm{th}} \approx 5 \mathrm{~mA}$ and $I \approx 11 \mathrm{~mA}$, only a few of these lasers switched polarization. Most of these polarization-switching devices exhibited hysteresis, because the switching occurred at relatively large currents, whereas some showed stochastic hopping since they switched at lower currents. To make a comparison with theory, very large numbers of VCSELs would be needed to end up with a reasonable number that switch at relatively low currents. Furthermore such a comparison is always hindered by unintentional differences from device to device. In order to solve this problem we used the socalled hot-spot technique [11] to vary the switch current of a single laser.

With this tool we changed the strength and orientation of the linear birefringence by applying stress with a focused 780-nm laser beam (typically $30 \mathrm{~mW}$ ) that locally heats the wafer surface next to the VCSEL. In experiments with the hot spot we have not noticed a direct effect on the dichroism, only an indirect one. By changing the position of the hot spot we can rotate the axes of the birefringence, so that the projected dichroism will change, assuming that linear birefringence is still the dominant anisotropy. By rotating the birefringence axes the projected dichroism, i.e., the first term in Eq. (2b), was canceled, which results in a bistable system with the laser hopping symmetrically between two linear polarizations determined by the axes of the linear birefringence. By adjustment of the hot-spot power the VCSEL can now be forced to hop at any current within the range of the fundamental transverse mode.

To measure the polarization of the emitted VCSEL light we use three different tools [2]: a Fabry-Pérot 
interferometer to measure the optical spectrum, a fast photodiode in combination with a spectrum analyzer to measure polarization-resolved intensity noise spectra (detection bandwidth $6 \mathrm{GHz}$ ) and a fast photodiode in combination with an oscilloscope to measure time traces (detection bandwidth $500 \mathrm{MHz}$ ).

The inset in Fig. 2 shows a typical time trace of the $x$-polarized part of the output power of a singletransverse-mode VCSEL that hops at $6.4 \mathrm{~mA}$. Spectral measurements with a Fabry-Pérot interferometer (not shown) confirmed that the two levels in the time trace corresponded with the two different linear VCSEL polarizations in the optical spectrum, only one of which was lasing at each time; from this we conclude that hopping occurs between the two polarizations in the fundamental transverse mode. A very long time trace, containing more than $10^{4}$ switches, was used to calculate the probability distribution of residence times, as shown in Fig. 2. This distribution is exponential, as expected from theory, with a fitted average residence time of $1.0 \mu \mathrm{s}$. We checked that the statistics of both levels in the time trace were the same.

Next we increased the current and adjusted the hot-spot power and/or position until the VCSEL hopped again. In this way the residence times were determined for different hop currents; the combined result is shown in Fig. 3. Note that each point corresponds with symmetric hopping. As one can see in Fig. 3, for low currents switching occurs on a submicrosecond time scale, whereas for higher currents the time scale rapidly increases to seconds. Such a huge change in the residence times (about 8 orders of magnitude) has never been observed before.

Measurements have been repeated for different VCSELs of our batch; the results shown in Fig. 3 are also representative for the other devices. An attempt to

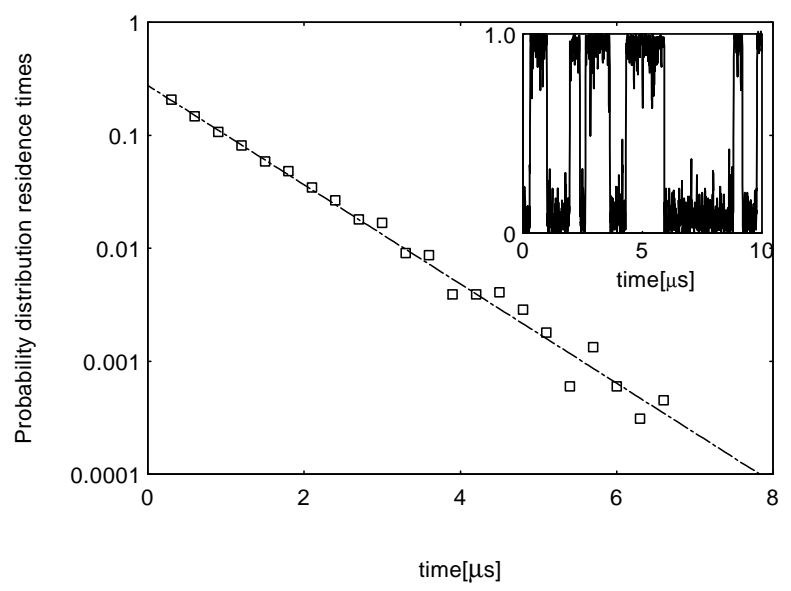

FIG. 2. Distribution of residence times of a polarization switching VCSEL at $I=6.4 \mathrm{~mA}$. From the exponential fit the average residence time was found to be $1.0 \mu \mathrm{s}$. The inset shows a small part of the polarization resolved time trace; the full trace was used to calculate the distribution. measure the time it takes for the polarization to actually switch gave an upper limit of $2 \mathrm{~ns}$. For the theory to be valid the inverse of this time must be much smaller than the linear birefringence $\omega_{\text {lin }}$, which causes the spiral motion during the switch [2]. As $\omega_{\text {lin }} \approx 15 \mathrm{~ns}^{-1}$ (see below) we satisfy this condition.

We will now discuss the independent measurements of both the deterministic and stochastic force which determine the residence time. First we have measured the effective anisotropies of the switching laser (Fig. 3) with polarization homodyne detection [2,9]. The nonlinear parts of the anisotropies (and thus also the linear parts) can be extracted from the discontinuities in the effective anisotropies around the switch [2], where the linear anisotropies change sign, but the nonlinear ones do not. (This is because linear anisotropies generate differences with respect to two orthogonal linear polarizations, whereas nonlinear anisotropies act the same on every linearly polarized state). As the switch current could be set with the hot spot, we could measure the nonlinear birefringence and nonlinear dichroism $(=$ depth of the potential well) as a function of $\mu=\left(I / I_{\text {th }}\right)-1$ (see Fig. 4). From the straight-line fits [Eq. (1c)] we determined the absolute strength of the nonlinear anisotropies (at $\mu=1$ ) as $\omega_{\text {non }}=4.5 \mathrm{~ns}^{-1}$ and $\gamma_{\mathrm{non}}=1.5 \mathrm{~ns}^{-1}$, which implies $\alpha \approx 3$ and $\Gamma \approx 200$, assuming the cavity decay rate to be $\kappa=300 \mathrm{~ns}^{-1}$. Furthermore we found that $\omega_{\text {lin }} \approx 15 \mathrm{~ns}^{-1}$, so that linear birefringence is indeed the dominant anisotropy, $\omega_{\operatorname{lin}} \gg \omega_{\text {non }}, \gamma_{\text {non }}$. The fitted threshold current in Fig. 4 was $5.1 \mathrm{~mA}$, which is slightly higher than the value of $4.8 \mathrm{~mA}$ determined from the output-input curve.

To measure the amount of spontaneous emission we have determined the optical linewidth with a selfheterodyne fiber-delay setup. The measured linewidth $\Delta \nu=\left(1+\alpha^{2}\right) D / \pi$ was about $20 \mathrm{MHz}$ at $\mu=1$, which corresponds with a diffusion rate $D^{\mu=1}$ of

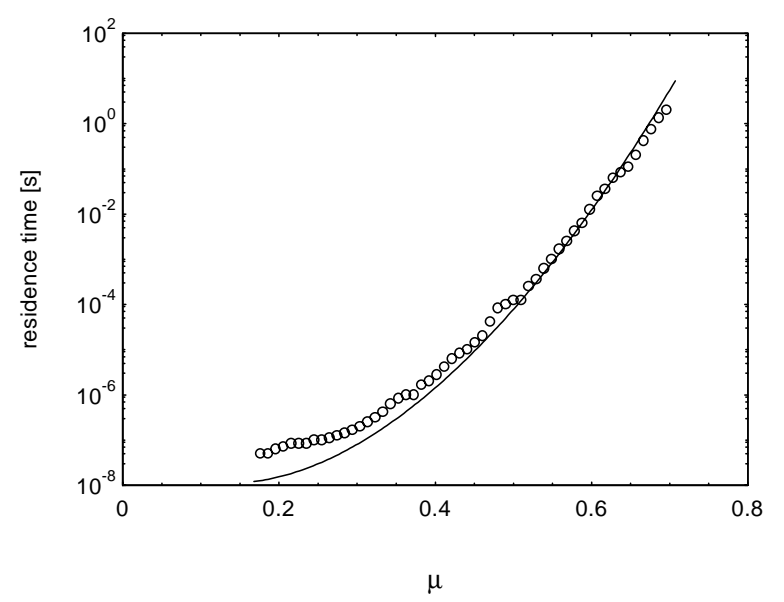

FIG. 3. Residence times as a function of the normalized pump parameter $\mu$; the dots are experimental data and the line is a theoretical fit corresponding to Eq. (3). 


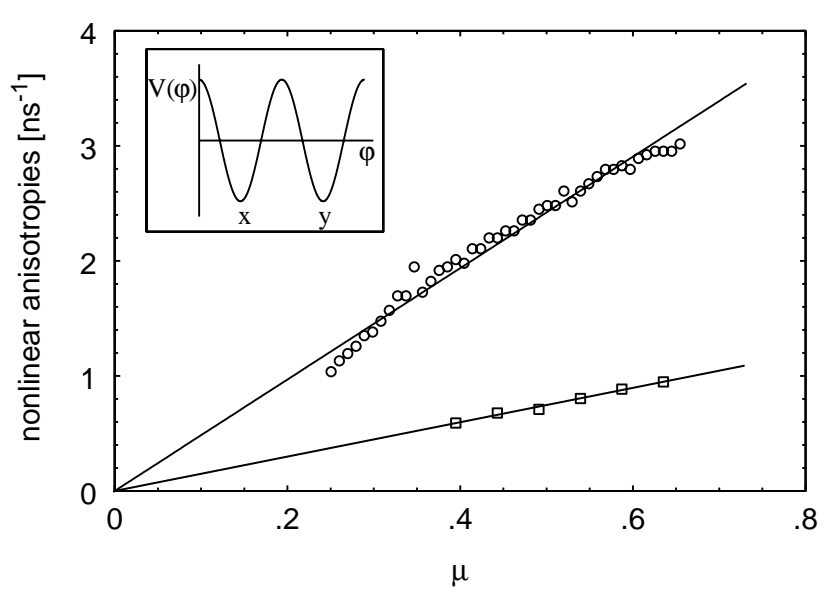

FIG. 4. Measured nonlinear birefringence $\omega_{\text {non }}$ (circles) and nonlinear dichroism $\gamma_{\mathrm{non}}$ (squares) as a function of the normalized pump $\mu$; the lines are theoretical fits [Eq. (1c)]. The inset shows a sketch of the potential; the barrier between two adjacent wells is proportional to $\gamma_{\text {non }}$.

7-12 $\mu \mathrm{s}^{-1}$, where the spread accounts for uncertainties in $\alpha$ and $\Delta \nu$.

These experimentally determined values of the potential well depth and the spontaneous emission noise are fully consistent with the fit values of $\kappa / \Gamma=1.5 \mathrm{~ns}^{-1}$ and $D^{\mu=1}=7.5 \mu \mathrm{s}^{-1}$ that we obtained when Eq. (3) was fitted to the data shown in Fig. 3. Note the good quality of the fit; we attribute the deviations for low pump values in Figs. 3 and 4 to the theoretical assumption that the relation between current and output power is linear, which is not really valid near threshold.

Our work has an interesting consequence for controlled polarization switching in VCSELs. In that case the double-well potential is made asymmetric by changing the laser current across a deterministic hop. In this case there are two parameters that determine the residence times: (i) the noise strength and (ii) the asymmetry of the potential set by the change in current. For polarization modulation it is preferable, for technical reasons, to have a small current-modulation depth. However, too small a modulation depth will not reduce the double well to a single well, leading to the response time and switch rate being limited by the amount of spontaneous emission noise. Furthermore, we note that the signal-to-noise ratio for polarization modulated devices depends on the noise strength in a similar way as in the so-called stochasticresonance phenomenon $[12,13]$.
In conclusion, we have experimentally studied symmetric polarization switching in VCSELs by changing their switch current with the hot-spot technique and thus tailoring the Kramers potential of an individual device. The results confirm the validity of a recently published analytical theory for the polarization of practical VCSELs [2].

We acknowledge support from the "Stichting voor Fundamenteel Onderzoek der Materie" (FOM), from the European Union under ESPRIT Project No. 20029 (ACQUIRE), and from TMR Network No. ERB4061PL951021 (Microlasers and Cavity QED).

[1] K. D. Choquette, R.P. Schneider, Jr., K. L. Lear, and R. E. Leibenguth, IEEE J. Sel. Top. Quantum Electron. 1, 661 (1995); C. J. Chang-Hasnain, J.P. Harbison, G. Hasnain, A.C. Von Lehmen, L. T. Florez, and N. G. Stoffel, IEEE J. Quantum Electron. 27, 1402 (1991); J. Martin-Regalado, J. L. A. Chilla, J. J. Rocca, and P. Brusenbach, Appl. Phys. Lett. 70, 3350 (1997); J. Martin-Regalado, S. Balle, and M. San Miquel, Opt. Lett. 22, 460 (1997); G. Giacomelli and F. Marin, Quantum Semiclass. Opt. 10, 469 (1998).

[2] M.P. van Exter, M. B. Willemsem, and J.P. Woerdman, Phys. Rev. A 58, 4191 (1998).

[3] H. A. Kramers, Physica (Utrecht) 7, 284 (1940).

[4] P. Hänggi, P. Talkner, and M. Borkovec, Rev. Mod. Phys. 62, 251 (1990); R. Roy, R. Short, J. Durnin, and L. Mandel, Phys. Rev. Lett. 45, 1486 (1980).

[5] M.P. van Exter, R.F.M. Hendriks, and J.P. Woerdman, Phys. Rev. A 57, 2080 (1998).

[6] M.P. van Exter, A. Al-Remawi, and J.P. Woerdman, Phys. Rev. Lett. 80, 4875 (1998).

[7] J. Martin-Regalado, F. Prati, M. San Miguel, and N. B. Abraham, IEEE J. Quantum Electron. 33, 765 (1997).

[8] M. San Miguel, Q. Feng, and J. V. Moloney, Phys. Rev. A 52, 1728 (1995).

[9] H. F. Hofmann and O. Hess, Quantum Semiclass. Opt. 10, 87 (1998).

[10] Vixel Corporation, 325 Interlocken Parkway, Broomfield, CO 80021, Model No. PRI-LA-S-850-1x8-3S.

[11] A. K. Jansen van Doorn, M. P. van Exter, J. P. Woerdman, Appl. Phys. Lett. 70, 1041-1043 (1996).

[12] B. McNamara, K. Wiesenfeld, and R. Roy, Phys. Rev. Lett. 60, 2626 (1988).

[13] After submission of our manuscript a paper has appeared, reporting on the observation of stochastic resonance in VCSELs: G. Giacomelli, F. Marin, and I. Rabbiosi, Phys. Rev. Lett. 82, 675 (1999). 